\title{
Aplicação de Probabilidades de Transição de Estado Dependentes do Tempo na Análise Quantitativa do Comportamento Ingestivo de Ovinos. Parte II ${ }^{1}$
}

\author{
Vívian Fischer², Pierre Dutilleul ${ }^{3}$, Armand Gerard Deswysen ${ }^{4}$, Lionel Dèspres ${ }^{4}$, \\ José Fernando Piva Lobato ${ }^{5}$
}

\begin{abstract}
RESUMO - Os padrões do comportamento ingestivo foram avaliados usando nove ovinos $1 / 2$ Texel +1/2 Ile-de-France durante seis meses. Os ovinos receberam a dieta às 9 e $16 \mathrm{~h}$, composta de $250 \mathrm{~g}$ de concentrado $(15,45 \% \mathrm{~PB}, 36,54 \%$ FDN) e feno de gramíneas à vontade $(6,69 \% \mathrm{~PB}, 69,10 \% \mathrm{FDN})$. O comportamento ingestivo foi medido continuamente durante cinco dias por período, suas atividades foram classificadas como ingestão, ruminação ou descanso eregistradas seqüencialmente. O cálculo das probabilidades de estado abrangeu três tipos: as probabilidades de estar em determinado estado, de permanecer neste estado e de mudar de um estado para outro. Foi aplicada a transformação finita de Fourier sobre as séries de probabilidades estimadas a cada cinco minutos, durante 22 horas por dia. No estudo da evolução nictemeral das probabilidades, foram utilizadas as análises de periodogramas, diagramas de fase e análise da variância sobre os valores transformados das séries de probabilidades. A análise de variância mostrou que todos os tipos de probabilidades seguiram uma distribuição nictemeral, influenciada significativamente pelos períodos e animais experimentais, em diversas frequiências. A análise dos periodogramas e diagramas de fase mostrou que as diferenças entre os períodos e animais experimentais foi especialmente devido às diferenças de amplitude das funções trigonométricas que descreveram a distribuição nictemeral das probabilidades de permanecer ou estar em determinado estado. O momento do dia, o período experimental e os indivíduos influenciaram a distribuição nictemeral das probabilidades de transição de estado.
\end{abstract}

Palavras-chave: diagramas de fase, periodogramas, transformação finita de Fourier

\section{Use of Time-Dependent Transition Probabilities for Quantitative Analysis of Ingestive Behavior of Sheep. Part II}

\begin{abstract}
The ingestive behavior patterns were evaluated in nine sheep 1/2 Texel + 1/2 Ile-de-France over a six month period. Sheep were fed the diet at 9 a.m. and 4 p.m., which was composed by $250 \mathrm{~g}$ of concentrate $(15.45 \%$ CP, $36.54 \%$ NDF) and ad libitum grass hay $(6.69 \% \mathrm{CP}, 69.10 \% \mathrm{NDF})$. Ingestive behavior was continuously measured for five days per period, and its activities or states were classified as eating, ruminating or idling, and sequentially registered. The probability estimates incorporated three types: The probabilities of being in a given state, of staying in a given state and of changing from a state to another one. Finite Fourier transformation was applied to the series of probabilities estimated at each five minutes, during 22 hours per day. In the nycterohemeral probabilities distribution study, it was used the analysis of periodgrams, phase diagrams and analysis of variance upon the transformed values of the probabilities series. The analysis of variance showed that all probability types followed a nycterohemeral distribution, and it was significantly affected by experimental period and animals at several frequencies. Analysis of periodgrams and phase diagrams showed that differences between the experimental periods and animals was mainly due to differences in the amplitude of trigonometric functions that described the nycterohemeral distribution of probabilities of being or staying in a given state. The day moment, the experimental period and individuals affected the nycterohemeral distribution of the transition state probabilities.
\end{abstract}

Key Words: finite Fourier transform, periodograms, phase diagrams

\section{Introdução}

A maioria dos trabalhos não apresenta uma análise quantitativa da distribuição do tempo despendido nas atividades ingestivas, e, até recentemente, os métodos quantitativos na análise comportamental de grande parte destes trabalhos basearam-se na aplicação dos métodos estatísticos univariados. Porém, são necessários métodos estatísticos avançados para permitir melhor compreensão dos fenômenos envolvidos nos estudos etológicos (COLGAN, 1978). Esta análise permite a identificação das diferentes fre-

\footnotetext{
1 Parte da tese de doutorado do primeiro autor, parcialmente financiada pelo CNPq e CAPES.

2 Departamento de Zootecnia, Faculdade de Agronomia, Universidade Federal de Pelotas, RS, Brasil. Bolsista do CNPq. E.mail: vfried@portoweb.com.br

3 Departamento de Fitotecnia, McGill University, Canadá. E.mail: dutilleul@musica.mcgill.ca

4 Departamento de Biologia Aplicada e Produção Animal, Université Catholique de Louvain, Bélgica. E.mail: deswysen@gena.ucl.ac.be

5 Departamento de Zootecnia, Faculdade de Agronomia, Universidade Federal do Rio Grande do Sul.
} 
quiências com que as atividades ingestivas ocorrem durante determinado intervalo de tempo e dos fatores que as afetaram. A análise quantitativa pode ser aplicada ao estudo da evolução nictemeral das probabilidades de transição de estado, constituindo-se em uma ferramenta no estudo do comportamento de ruminantes (DUTILLEUL et al., 2000).

Dois tipos de abordagem estatística podem ser utilizados: no âmbito temporal, com base na análise da variância de medidas repetidas, e no âmbito de frequiências, baseado nas análises de periodogramas e de diagramas de fase e na análise da variância da transformação finita de Fourier (DUTILLEUL, 1997). As técnicas para análise de séries temporais podem ser muito úteis na investigação das características não determinísticas do comportamento animal, em particular na análise dos dados comportamentais obtidos de forma seqüencial. A informação obtida por meio destas análises pode contribuir para esclarecer as relações entre diferentes tipos de atividades ou estados, a causa de determinado comportamento e as interações interindividuais (FAGEN e YOUNG, 1978).

A análise de periodogramas é um método chave no estudo estatístico realizado no âmbito das frequiências, sendo utilizado para a detecção de sinais periódicos. A idéia básica do uso do periodograma é que, quanto maior a amplitude de uma função co-seno ajustada à uma série temporal pelo método dos quadrados mínimos, maior será o valor do periodograma no período ou na freqüência correspondente (DUTILLEUL, 1990). A avaliação do periodograma de Schuster é normalmente limitada às frequiências de Fourier, que correspondem à média temporal (freqüência $=0$, zero ciclos) ou a um número inteiro de ciclos durante o intervalo de tempo considerado, variando de um até metade do valor do intervalo de tempo considerado. A análise dos diagramas de fase permite detectar a defasagem entre os diversos níveis de tratamentos ou variáveis classificatórias quanto à sua ocorrência em relação ao momento inicial em que os parâmetros comportamentais foram medidos. Dessa forma, a defasagem dos picos de máxima atividade ingestiva ou de ruminação em relação ao momento inicial do ciclo (fixado como o horário do primeiro arraçoamento ou do início dos registros do comportamento ingestivo) pode ser obtida pelos diagramas de fase (DESWYSEN et al. 1989).

A análise de variância dos dados modificados por meio da aplicação da transformação finita de Fourier permite a avaliação das diferenças entre os níveis dos fatores estudados no âmbito das freqüências
(BRILLINGER, 1973; DUTILLEUL, 1990). Esta análise da variância completa a análise do periodograma e do diagrama de fase, fornecendo uma sequiência de testes $F$ realizados nas freqüências de Fourier, para testar se as variáveis de interesse na forma de séries temporais diferem entre grupos ou níveis dos fatores estudados quanto à sua amplitude ou fase. Esta seqüência de testes resulta da decomposição ortogonal da interação tratamento x tempo por intemédio da aplicação da transformação finita de Fourier. Após a sua aplicação, o modelo usado na análise de variância é bivariado em todas as frequiências, exceto na frequiência zero, e os efeitos testados são aqueles relativos aos tratamentos e às variáveis classificatórias. A interpretação biológica das partes real e imaginária da transformação finita de Fourier é facilitada pela sua reparametrização em amplitude e fase para cada uma das freqüências utilizadas. As diferenças estatísticas entre os diversos fatores estudados para cada componente de ritmo ou frequiência podem ser explicadas pelas diferenças quanto à amplitude, representada pelo periodograma, e, ou, à fase representada pelo diagrama de fase (DESWYSEN et al., 1989).

As análises quantitativas no âmbito das freqüências foram aplicadas às séries temporais dos tempos gastos em atividades pertencentes ao repertório do estudo comportamental em diversas espécies, como bovinos de leite (BEAUCHEMIN et al., 1990; DESWYSEN et al., 1993), bovinos de corte (FISCHER, 1996), ovinos em pastejo (FISCHER et al., 1998a) e confinados (FISCHER et al., 1998b), mas foram relativamente pouco aplicadas no estudo probabilístico de parâmetros do comportamento. Nesse caso, citam-se poucos trabalhos, como o estudo do comportamento de ovinos (FISCHER, 1996; DUTILLEUL et al., 2000).

O objetivo do presente estudo foi a avaliação da distribuição nictemeral das probabilidades de transição de estado dependente do tempo aplicadas ao estudo do comportamento de ovinos.

\section{Material e Métodos}

O experimento foi desenvolvido entre outubro de 1992 e abril de 1993 e consistiu de seis períodos de 28 dias cada um. Os períodos de 1 a 6 correspondem, respectivamente, aos meses de outubro, novembro, dezembro, janeiro, fevereiro e março/abril.

Os animais utilizados foram ovinos $1 / 2$ Texel+ 1/2 Ile de France, sendo seis fêmeas e três machos 
Rev. bras. zootec.

castrados, com aproximadamente oito meses de idade e peso vivo médio inicial de 52,1+6,2 kg. Os animais receberam a mesma dieta durante todo o experimento, distribuída duas vezes ao dia (às 9 e 16h), que consistiu de $250 \mathrm{~g}$ de concentrado com $15,45 \%$ de proteína bruta (PB) e 36,54\% de fibra em detergente neutro (FDN), além de feno de gramíneas temperadas à vontade, com 6,69\% PB e 69,10\% FDN, permitindo-se 15 a $20 \%$ de sobras. Os ingredientes que compuseram o concentrado usado foram: $18 \%$ torta de linho, 2,25\% torta de algodão, 3,15\% farelo de soja, 6,30\% feno de alfafa, 2,70\% melaço, 3,6\% suplemento mineral e vitamínico, $32,50 \%$ flocos de cevada, $31,50 \%$ de polpa. Sal mineral e água estiveram permanentemente disponíveis.

O comportamento ingestivo foi registrado continuamente, durante cinco dias em cada período experimental, segundo o método descrito por RUCKEBUSH (1963). Gerou-se uma lista seqüencial com os tempos de ingestão, ruminação e descanso para cada combinação de animal $(n=9)$, dia $(n=5)$ e período $(n=6)$. Neste estudo, foram utilizados os registros das primeiras 22 horas em cada dia, em virtude da perda de registros de alguns animais ocorrida nas últimas horas. As 22 horas de registros representaram cerca de $92 \%$ do período total, sendo possível estimar padrões nictemerais de probabilidades a partir destes dados.

Foram calculadas séries de probabilidades de estado a partir de três situações: estar em determinado estado ou atividade ingestiva, permanecer em determinado estado ou mudar de um estado para outro (DUTILLEUL et al., 2000). Estes cálculos se basearam no mesmo princípio estatístico de se estimar a probabilidade de um evento em relação a outros possíveis, por meio da freqüência relativa observada (SNEDECOR e COCHRAN, 1967). Os cálculos foram efetuados a cada 5 minutos e os cinco dias de medida do comportamento ingestivo, dentro de cada período, foram usados como repetições. Os cálculos foram detalhados em DUTILLEUL et al. (2000). As probabilidades de estar (PEE) ou permanecer em determinado estado (PPE) ou mudar de um estado para outro (PME) podem ser escritas matematicamente como: $\mathrm{PEE} a=\mathrm{P}($ estado $a$ tempo $t)$; PPE $a t=\mathrm{P}($ estado $a$ tempo $t \cap$ estado $a$ tempo $t+\Delta t)$; PMEabt $=\mathrm{P}$ (estado $a$ tempo $t \cap$ estado $b$ tempo $t+\Delta t$ ), em que $\mathrm{P}$ é medida clássica de probabilidade e a, estado comportamental ( $\mathrm{a}=1,2$ ou 3 ).

As séries temporais de probabilidades de estar ou, permanecer em determinado estado e de transi- ção de estado foram submetidas à transformação finita de Fourier (TFF). Foi realizada a análise de variância destes dados transformados para averiguar os efeitos dos diferentes períodos de medida e da variação entre os animais, segundo o modelo:

$$
Y_{i j k}=U+A_{i}+P_{j}+A P_{i j}+E_{i j k}
$$

em que $\mathrm{U}$ é média geral; $\mathrm{A}_{\mathrm{i}}$, efeito animal $(\mathrm{GL}=8)$; $P_{j}$, efeito período $(G L=5) ; A P_{i j}$, efeito da interação entre animale período $(\mathrm{GL}=40) ; \mathrm{e}_{\mathrm{ijk}}$, erro experimental $(\mathrm{GL}=0)$.

Os métodos estatísticos foram detalhados em DESWYSEN et al. (1989). No presente estudo, o número de ciclos em 22 horas ou freqüências variou entre zero (média geral) e 132, porém somente os resultados relativos às primeiras 12 freqüências serão apresentados, por serem os mais significativos. As hipóteses de nulidade testadas foram rejeitadas, quando o valor de probabilidade foi igual ou menor que 0,05. Estas análises foram realizadas por intermédio do procedimento GLM do SAS (1989) e SPECTRA do SAS (1988). As causas das diferenças entre os níveis das variáveis classificatórias (período e animal) detectadas pela análise da variância da TFF, se decorrentes da diferença de amplitude ou defasagem no tempo de ocorrência em relação ao início dos registros do comportamento, foram realizadas por meio da inspeção dos valores das ordenadas de periodogramas e diagramas de fase.

\section{Resultados e Discussão}

A evolução nictemeral da possibilidade de permanecer e estar ingerindo, ruminando e descansando apresentou diferenças significativas entre os períodos de medidas e entre os animais (Tabelas 1 e 2). A discussão dos resultados ficará restrita ao estudo das probabilidades de estar em determinado estado, pois os resultados obtidos para as probabilidades de permanecer em cada um dos três estados foram muito semelhantes àqueles para as probabilidades de estar em um dos três estados.

Considerando o estado de ingestão, a ausência de efeito principal de período foi rejeitada no nível de 0,05 em nove das 12 primeiras frequiências de Fourier: freqüência 2, que define um dos componentes fundamentais do ritmo nas fases diurna e noturna do dia e é representado por uma função trigonométrica com ciclos a cada 11 horas, e freqüências $3,4,5,6,7,9$, 11 e 12, que são harmônicas em relação à frequiência 2 e correspondem, respectivamente, a ciclos de 7,33; 5,$5 ; 4,4 ; 3,67 ; 3,14 ; 2,44 ; 2$ e 1,83 horas (Tabela 2). 
FISCHER et al.

Tabela 1 - Valores das probabilidades mínimas de rejeição da hipótese de nulidade dos efeitos período e animal obtidos a partir da análise da variância das séries de probabilidades de permanência nos estados de ingestão, descanso e ruminação, modificadas pela transformação finita de Fourier em cada freqüência.

Table 1 - Minimal probabilities values of null hypothesis rejection of no animal or period effect obtained from variance analysis of probablilities series of staying eating, idling or ruminating modified through finite Fourier transformation at each frequency

\begin{tabular}{|c|c|c|c|c|c|c|}
\hline \multirow{4}{*}{$\begin{array}{l}\text { Freqüência } \\
\text { Frequency }\end{array}$} & \multicolumn{6}{|c|}{$\begin{array}{c}\text { Valores de } \mathrm{P} \\
P \text { values }\end{array}$} \\
\hline & \multicolumn{2}{|c|}{$\begin{array}{l}\text { Ingestão } \\
\text { Eating }\end{array}$} & \multicolumn{2}{|c|}{$\begin{array}{l}\text { Descanso } \\
\text { Idling }\end{array}$} & \multicolumn{2}{|c|}{$\begin{array}{l}\text { Ruminação } \\
\text { Ruminating }\end{array}$} \\
\hline & Animal & Período & Animal & Período & Animal & Período \\
\hline & Animal & Period & Animal & Period & Animal & Period \\
\hline $0^{1}$ & 0,001 & 0,296 & 0,001 & 0,109 & 0,005 & 0,354 \\
\hline 1 & 0,001 & 0,065 & 0,001 & 0,001 & 0,001 & 0,002 \\
\hline 2 & 0,120 & 0,001 & 0,022 & 0,017 & 0,020 & 0,696 \\
\hline 3 & 0,010 & 0,001 & 0,001 & 0,392 & 0,001 & 0,018 \\
\hline 4 & 0,617 & 0,004 & 0,056 & 0,132 & 0,749 & 0,013 \\
\hline 5 & 0,255 & 0,001 & 0,645 & 0,311 & 0,173 & 0,037 \\
\hline 6 & 0,082 & 0,005 & 0,001 & 0,046 & 0,287 & 0,022 \\
\hline 7 & 0,030 & 0,012 & 0,233 & 0,027 & 0,373 & 0,240 \\
\hline 8 & 0,342 & 0,036 & 0,106 & 0,539 & 0,781 & 0,239 \\
\hline 9 & 0,039 & 0,020 & 0,247 & 0,083 & 0,245 & 0,906 \\
\hline 10 & 0,014 & 0,127 & 0,745 & 0,267 & 0,434 & 0,147 \\
\hline 11 & 0,003 & 0,008 & 0,629 & 0,599 & 0,845 & 0,420 \\
\hline 12 & 0,716 & 0,002 & 0,057 & 0,250 & 0,177 & 0,605 \\
\hline
\end{tabular}

1 Valor da probabilidade de rejeição da hipótese de nulidade obtida da análise da variância para a média geral das probabilidades de permanecer ingerindo, descansando ou ruminando.

1 Value of null hypothesis rejection obtained from variance analysis of overall mean probability of staying eating, idling and ruminating.

Tabela 2 - Valores das probabilidades mínimas de rejeição da hipótese de nulidade dos efeitos período e animal obtidos a partir da análise da variância das séries de probabilidades de estar nos estados de ingestão, descanso e ruminação, modificadas pela transformação finita de Fourier em cada freqüência

Table 2 - Minimal probabilities values of null hypothesis rejection of no animal or period effect obtained from variance analysis of probablilities series of being eating, idling or ruminating modified through finite Fourier transformation at each frequency

\begin{tabular}{|c|c|c|c|c|c|c|}
\hline \multirow{4}{*}{$\begin{array}{l}\text { Freqüência } \\
\text { Frequency }\end{array}$} & \multicolumn{6}{|c|}{$\begin{array}{c}\text { Valores de } \mathrm{P} \\
P \text { values }\end{array}$} \\
\hline & \multicolumn{2}{|c|}{$\begin{array}{l}\text { Ingestão } \\
\text { Eating }\end{array}$} & \multicolumn{2}{|c|}{$\begin{array}{l}\text { Descanso } \\
\text { Idling }\end{array}$} & \multicolumn{2}{|c|}{$\begin{array}{c}\text { Ruminação } \\
\text { Ruminating }\end{array}$} \\
\hline & Animal & Período & Animal & $\overline{\text { Período }}$ & Animal & Período \\
\hline & Animal & Period & Animal & Period & Animal & Period \\
\hline $0^{1}$ & 0,001 & 0,372 & 0,001 & 0,127 & 0,008 & 0,329 \\
\hline 1 & 0,001 & 0,063 & 0,001 & 0,001 & 0,001 & 0,008 \\
\hline 2 & 0,063 & 0,001 & 0,016 & 0,010 & 0,214 & 0,595 \\
\hline 3 & 0,005 & 0,001 & 0,001 & 0,600 & 0,001 & 0,005 \\
\hline 4 & 0,781 & 0,005 & 0,036 & 0,179 & 0,648 & 0,007 \\
\hline 5 & 0,379 & 0,001 & 0,638 & 0,100 & 0,167 & 0,064 \\
\hline 6 & 0,109 & 0,002 & 0,001 & 0,033 & 0,145 & 0,033 \\
\hline 7 & 0,033 & 0,008 & 0,102 & 0,053 & 0,288 & 0,104 \\
\hline 8 & 0,334 & 0,081 & 0,155 & 0,622 & 0,726 & 0,371 \\
\hline 9 & 0,031 & 0,017 & 0,255 & 0,068 & 0,176 & 0,857 \\
\hline 10 & 0,018 & 0,075 & 0,628 & 0,240 & 0,367 & 0,113 \\
\hline 11 & 0,004 & 0,021 & 0,682 & 0,467 & 0,844 & 0,507 \\
\hline 12 & 0,688 & 0,006 & 0,104 & 0,261 & 0,172 & 0,545 \\
\hline
\end{tabular}

1 Valor da probabilidade de rejeição da hipótese de nulidade obtida da análise da variância para a média geral das probabilidades de estar ingerindo, descansando ou ruminando.

${ }^{1}$ Value of null hypothesis rejection obtained from variance analysis of overall mean probability of being eating, idling and ruminating. 
Rev. bras. zootec.

O efeito principal significativo de período nas nove frequiências de Fourier para o estado de ingestão é caracterizado principalmente por diferenças nos valores da ordenada do periodograma entre os períodos, especialmente nas frequiências 3, 4, 6 e 7 (Tabela 3).

As diferenças nos valores do periodograma indicam que as frequiências 3 e 4 apresentaram maiores amplitudes nos períodos março/abril e outubro em comparação a janeiro, enquanto os componentes de ritmo de maior frequiência (como por exemplo, 6, 9, 12) apresentaram maiores amplitudes em janeiro em relação a março/abril. Estes resultados confirmam a análise visual realizada por meio da inspeção de gráficos da distribuição nictemeral das probabilidades de estar ingerindo (FISCHER et al., 2000), quando se constatou a ocorrência de picos de maior amplitude da probabilidade estar ingerindo em pelo menos três momentos do nictêmero (após as refeições principais e no início da noite) em março/abril, em comparação com janeiro. Não houve diferenças entre os períodos quanto aos valores do diagrama de fase, significando que, em média, não houve efeito do fotoperíodo sobre o momento de ocorrência dos valores máximos para as probabilidades de estar ingerindo.

Em relação ao efeito principal animal, constatou-se que ocorreram diferenças significativas entre os animais em seis das 12 primeiras freqüências de Fourier: frequiência 1 e suas harmônicas 3, 7, 9, 10 e 11
(Tabela 2), principalmente relacionadas às diferenças de amplitude das funções trigonométricas ajustadas às curvas de distribuição nictemeral das probabilidades de estar no estado de ingestão (Tabela 4).

Os animais números 2 e 4 apresentaram, respectivamente, valores extremos de consumo voluntário de matéria seca, 44,29 e 55,43 g/kg PV $0,75 /$ dia (FISCHER, 1996). Os componentes de menor freqüência (1 e 3) apresentaram maiores amplitudes para o animal 2 (menor consumo) comparado com o animal 4 (maior consumo). Ocorreu o contrário em relação aos componentes de ritmo de maior freqüência, 7, 9 e 10. Estes resultados concordam com a análise visual dos gráficos da distribuição nictemeral das probabilidades de transição de estado (FISCHER, 1996), na qual foi observado que o animal com maior consumo voluntário apresentou maior probabilidade de estar ingerindo entre 9 e $21 \mathrm{~h}$.

Considerando o estado de ruminação, a ausência de efeito principal de período foi rejeitada no nível de 0,05 em 4 das 12 primeiras freqüências de Fourier: freqüência 1 , que define o componente fundamental do ritmo nictemeral e é representado por uma função trigonométrica com ciclos a cada 22 horas; e freqüências 3, 4 e 6, que são harmônicas em relação à freqüência 1 e correspondem, respectivamente, a ciclos de 7,33; 5,5; e 3,67 horas (Tabela 2).

$\mathrm{O}$ efeito principal significativo de período nas freqüências de Fourier, para a probabilidade de estar

Tabela 3 - Valores das ordenadas do periodograma relativo à probabilidade de estar ingerindo, segundo os períodos experimentais nas 12 primeiras freqüências de Fourier

Table 3 - Periodogram ordinates values for probability of being eating, according to the experimental periods at the first twelve Fourier frequencies

\begin{tabular}{|c|c|c|c|c|c|c|}
\hline \multirow[b]{3}{*}{$\begin{array}{l}\text { Freqüência } \\
\text { Frequency }\end{array}$} & \multicolumn{6}{|c|}{$\begin{array}{c}\text { Ingestão } \\
\text { Eating }\end{array}$} \\
\hline & \multicolumn{6}{|c|}{$\begin{array}{l}\text { Períodos experimentais } \\
\text { Experimental periods }\end{array}$} \\
\hline & $\begin{array}{l}\text { Outubro } \\
\text { October }\end{array}$ & $\begin{array}{l}\text { Novembro } \\
\text { November }\end{array}$ & $\begin{array}{l}\text { Dezembro } \\
\text { December }\end{array}$ & $\begin{array}{l}\text { Janeiro } \\
\text { January }\end{array}$ & $\begin{array}{l}\text { Fevereiro } \\
\text { February }\end{array}$ & $\begin{array}{l}\text { Março/abril } \\
\text { March/April }\end{array}$ \\
\hline 1 & 1,7669 & 1,2798 & 1,1025 & 1,7156 & 2,8711 & 1,4052 \\
\hline 2 & 0,1654 & 0,5572 & 1,2872 & 1,0542 & 0,9817 & 0,7642 \\
\hline 3 & 3,6231 & 2,2417 & 2,2560 & 1,2239 & 2,9875 & 3,5593 \\
\hline 4 & 2,3158 & 3,0563 & 3,4266 & 1,6747 & 3,5681 & 3,7941 \\
\hline 5 & 0,4841 & 0,2577 & 0,1315 & 0,1491 & 0,9676 & 0,0321 \\
\hline 6 & 1,3561 & 0,9504 & 1,9833 & 1,8643 & 0,7807 & 1,7176 \\
\hline 7 & 0,6620 & 1,2042 & 1,2740 & 1,4443 & 0,8257 & 2,2076 \\
\hline 8 & 0,1690 & 0,0725 & 0,2491 & 0,1492 & 0,8883 & 0,2044 \\
\hline 9 & 0,6745 & 0,3708 & 0,5514 & 0,3696 & 0,0846 & 0,1834 \\
\hline 10 & 0,5910 & 0,6976 & 0,4635 & 0,4746 & 0,7161 & 1,0321 \\
\hline 11 & 0,0479 & 0,1631 & 0,0871 & 0,0372 & 0,4030 & 0,0929 \\
\hline 12 & 0,1791 & 0,1012 & 0,0580 & 0,0826 & 0,0011 & 0,0239 \\
\hline
\end{tabular}


FISCHER et al.

Tabela 4 - Valores das ordenadas do periodograma relativo à probabilidade de estar ingerindo segundo os animais experimentais nas 12 primeiras freqüências de Fourier

Table 4 - Periodogram ordinates values for probability of being eating, according to the experimental animals at the first twelve Fourier frequencies

\begin{tabular}{|c|c|c|c|c|c|c|c|c|c|}
\hline \multirow[b]{3}{*}{$\begin{array}{l}\text { Freqüência } \\
\text { Frequency }\end{array}$} & \multicolumn{8}{|c|}{$\begin{array}{l}\text { Ingestão } \\
\text { Eating }\end{array}$} & \multirow[b]{3}{*}{9} \\
\hline & \multicolumn{8}{|c|}{$\begin{array}{c}\text { Animais experimentais } \\
\text { Experimental animals }\end{array}$} & \\
\hline & 1 & 2 & 3 & 4 & 5 & 6 & 7 & 8 & \\
\hline 1 & 1,5175 & 1,9461 & 1,8691 & 0,9148 & 2,4684 & 1,6351 & 1,2273 & 3,3917 & 0,9056 \\
\hline 2 & 0,5865 & 0,5413 & 0,8399 & 1,0916 & 0,8318 & 1,2983 & 0,3871 & 0,9117 & 0,4195 \\
\hline 3 & 1,9793 & 2,8109 & 1,3813 & 2,1015 & 2,3411 & 3,0553 & 4,0282 & 2,6040 & 3,3849 \\
\hline 4 & 2,5199 & 2,8745 & 1,9693 & 2,6801 & 2,8955 & 3,5469 & 3,4545 & 3,4279 & 2,8044 \\
\hline 5 & 0,2626 & 0,2592 & 0,2388 & 0,0431 & 0,0452 & 0,5086 & 0,3093 & 0,3402 & 0,1183 \\
\hline 6 & 0,8199 & 1,0804 & 0,7469 & 1,2844 & 1,8241 & 1,9332 & 1,5449 & 1,8937 & 1,6814 \\
\hline 7 & 0,8787 & 0,9375 & 0,5387 & 1,5889 & 2,1520 & 1,0585 & 1,1382 & 1,2974 & 1,9203 \\
\hline 8 & 0,4981 & 0,4904 & 0,3109 & 0,0957 & 0,1886 & 0,3665 & 0,1562 & 0,0340 & 0,4882 \\
\hline 9 & 0,3017 & 0,3629 & 0,2113 & 0,7246 & 0,5001 & 0,2649 & 0,3401 & 0,6048 & 0,0997 \\
\hline 10 & 0,6190 & 0,6096 & 0,5844 & 0,6880 & 1,5949 & 0,5783 & 0,0202 & 0,7082 & 0,7947 \\
\hline 11 & 0,2990 & 0,4527 & 0,5065 & 0,0209 & 0,0551 & 0,1717 & 0,0434 & 0,0090 & 0,0756 \\
\hline 12 & 0,1134 & 0,0105 & 0,0107 & 0,0244 & 0,0269 & 0,0161 & 0,1042 & 0,0953 & 0,0766 \\
\hline
\end{tabular}

no estado de ruminação, foi caracterizado por diferenças nos valores da ordenada do periodograma entre os períodos, nas quatro freqüências anteriormente citadas (Tabela 5). As diferenças nos valores do periodograma indicam que as freqüências 3 e 4 apresentaram maior amplitude em março/abril, em comparação a janeiro, enquanto as freqüências 1 e 6 , maiores amplitudes em janeiro em relação a março/ abril. Estes resultados confirmam a análise visual realizada por intermédio da inspeção de gráficos da distribuição nictemeral das probabilidades de estar ruminando (FISCHER et al., 2000), quando se constatou a influência do ritmo de alimentação sobre as demais atividades do repertório comportamental, confirmando observações anteriores de DESWYSEN et al. (1989) de que a ingestão e a ruminação competem pela alocação do tempo gasto pelo animal.

A freqüência 3 apresentou diferenças de fase

Tabela 5 - Valores das ordenadas do periodograma relativos à probabilidade de estar ruminando, segundo os períodos experimentais nas 12 primeiras freqüências de Fourier

Table 5 - Periodogram ordinates values for probability of being ruminating according to experimental periods at the first twelve Fourier frequencies

\begin{tabular}{|c|c|c|c|c|c|c|}
\hline \multirow{4}{*}{$\begin{array}{l}\text { Freqüência } \\
\text { Frequency }\end{array}$} & \multicolumn{6}{|c|}{$\begin{array}{l}\text { Ruminação } \\
\text { Ruminating }\end{array}$} \\
\hline & \multicolumn{6}{|c|}{$\begin{array}{c}\text { Períodos experimentais } \\
\text { Experimental periods }\end{array}$} \\
\hline & Outubro & Novembro & Dezembro & Janeiro & Fevereiro & Março/abril \\
\hline & October & November & December & January & February & March/April \\
\hline 1 & 0,8338 & 0,7054 & 0,7468 & 0,5048 & 0,6676 & 0,0838 \\
\hline 2 & 0,1403 & 0,2810 & 0,3877 & 0,2867 & 0,2299 & 0,2568 \\
\hline 3 & 2,0871 & 1,4345 & 1,7412 & 0,6616 & 2,5203 & 2,2242 \\
\hline 4 & 0,6743 & 1,6149 & 1,7397 & 1,2260 & 1,6825 & 2,1780 \\
\hline 5 & 0,4473 & 0,1893 & 0,1343 & 0,0460 & 0,2624 & 0,0243 \\
\hline 6 & 0,2549 & 0,3552 & 0,6765 & 0,9952 & 0,6292 & 0,6598 \\
\hline 7 & 0,3128 & 0,5846 & 0,1910 & 0,3577 & 0,1792 & 0,8306 \\
\hline 8 & 0,0194 & 0,0973 & 0,1157 & 0,1167 & 0,4597 & 0,1000 \\
\hline 9 & 0,2025 & 0,0904 & 0,0418 & 0,0664 & 0,3306 & 0,0124 \\
\hline 10 & 0,3134 & 0,1157 & 0,2667 & 0,3272 & 0,0407 & 0,2653 \\
\hline 11 & 0,0716 & 0,0730 & 0,0748 & 0,0067 & 0,1217 & 0,0538 \\
\hline 12 & 0,0039 & 0,0138 & 0,0108 & 0,1615 & 0,0773 & 0,0678 \\
\hline
\end{tabular}


Rev. bras. zootec.

entre os períodos de medida. Nesta frequiência, os valores de diagrama de fase foram 41,39, 38, 0, $39 \mathrm{e}$ 37 minutos, respectivamente para os períodos outubro, novembro, dezembro, janeiro, fevereiro e março/ abril. O pico da probabilidade de estar ruminando foi atingido entre 37 e 40 minutos mais cedo em janeiro em relação aos demais meses. Entretanto, as demais freqüências não apresentaram diferenças de fase entre os períodos de medida. Por outro lado, ZINN et al. (1983) observaram a antecipação da ocorrência das refeições, quando os animais foram submetidos a fotoperíodos curtos naturalmente ou não. Os ovinos submetidos a menores comprimentos do dia anteciparam o início da atividade de ruminação à tarde e à noite, como foi constatado anteriormente por FISCHER et al. (1998b).

Em relação ao efeito principal animal, constatou-se que ocorreram diferenças significativas entre os animais em duas das 12 primeiras frequiências de Fourier: frequiências 1 e 3 (Tabela 2), relacionadas às diferenças de amplitude das funções trigonométricas ajustadas às curvas de distribuição nictemeral das probabilidades de estar ruminando (Tabela 6). Quando se compararam os animais 2 e 4 , verificou-se que as freqüências 1 e 3 apresentaram maiores amplitudes para o animal 2. Estes resultados concordam com a inspeção visual dos gráficos da distribuição nictemeral das probabilidades de transição de estado (FISCHER, 1996), na qual foi observado que o animal com maior consumo voluntário apresentou maior probabilidade de estar ruminando entre 9 e $22 \mathrm{~h}$.

Para o estado de descanso, a ausência de efeito principal de período foi rejeitada no nível de $0,05 \mathrm{em}$ três das 12 primeiras freqüências de Fourier: freqüência 1, que corresponde a um ciclo a cada 22 horas, e freqüências 2 e 6 , que são harmônicas em relação à frequiência 1 e correspondem, respectivamente, a ciclos de 11 e 3,67 horas (Tabela 2).

O efeito principal significativo de período nas três freqüências de Fourier para o estado de descanso é caracterizado por diferenças nos valores da ordenada do periodograma entre os períodos, nas freqüências 1,2 e 6 (Tabela 7). As diferenças nos valores do periodograma indicam que as freqüências 1 e 2 apresentaram maiores amplitudes em fevereiro em comparação aos demais, enquanto a freqüência 6 apresentou maior amplitude em dezembro em relação aos outros meses.

Entretanto, a análise dos diagramas de fase mostra que a freqüência 1 apresentou valores diferentes entre os períodos, de $0,117,113,110,107$ e 98 minutos, respectivamente para os períodos de outubro, novembro, dezembro, janeiro e março/abril. No período de outubro, o pico da probabilidade de estar descansando ocorreu 98 a 117 minutos antes dos demais meses.

Em relação ao efeito principal animal, constatou-se que houve diferenças significativas entre os animais

Tabela 6 - Valores das ordenadas do periodograma relativo à probabilidade de estar ruminando segundo os animais experimentais nas 12 primeiras freqüências de Fourier

Table 6 - Periodogram ordinates values for probability of being ruminating according to experimental animals at the first twelve Fourier frequencies

\begin{tabular}{|c|c|c|c|c|c|c|c|c|c|}
\hline \multirow[b]{3}{*}{$\begin{array}{l}\text { Freqüência } \\
\text { Frequency }\end{array}$} & \multicolumn{8}{|c|}{$\begin{array}{l}\text { Ruminação } \\
\text { Ruminating }\end{array}$} & \multirow[b]{3}{*}{9} \\
\hline & \multicolumn{8}{|c|}{$\begin{array}{c}\text { Animais experimentais } \\
\text { Experimental animals }\end{array}$} & \\
\hline & 1 & 2 & 3 & 4 & 5 & 6 & 7 & 8 & \\
\hline 1 & 1,3287 & 0,6650 & 1,0635 & 0,4979 & 0,7140 & 0,0348 & 0,0245 & 2,3284 & 0,2957 \\
\hline 2 & 0,1945 & 0,0467 & 0,8665 & 0,5781 & 0,2433 & 0,3661 & 0,1549 & 0,4383 & 0,0538 \\
\hline 3 & 3,4436 & 3,4571 & 1,2222 & 0,8453 & 1,2622 & 1,6932 & 2,0769 & 0,7801 & 2,5174 \\
\hline 4 & 2,0369 & 1,7726 & 1,3075 & 1,2494 & 1,1734 & 1,2208 & 1,7761 & 1,1001 & 1,5063 \\
\hline 5 & 0,2745 & 0,1733 & 0,3853 & 0,0053 & 0,0192 & 0,2610 & 0,1457 & 0,3120 & 0,1602 \\
\hline 6 & 0,6743 & 0,4651 & 0,4663 & 0,5877 & 0,2214 & 0,6094 & 0,6716 & 0,4178 & 1,3041 \\
\hline 7 & 0,2428 & 0,0139 & 0,1975 & 0,6407 & 0,7974 & 0,2468 & 0,6346 & 0,5600 & 0,5881 \\
\hline 8 & 0,0125 & 0,0966 & 0,1698 & 0,0351 & 0,0853 & 0,6212 & 0,1432 & 0,0923 & 0,1519 \\
\hline 9 & 0,1700 & 0,1484 & 0,2509 & 0,3084 & 0,4095 & 0,0791 & 0,0207 & 0,2255 & 0,1354 \\
\hline 10 & 0,1278 & 0,0980 & 0,1152 & 0,2453 & 0,2823 & 0,0256 & 0,0271 & 0,5071 & 0,5774 \\
\hline 11 & 0,0032 & 0,1128 & 0,3367 & 0,0510 & 0,0040 & 0,0151 & 0,0742 & 0,0712 & 0,0438 \\
\hline 12 & 0,1212 & 0,0820 & 0,0160 & 0,0628 & 0,1691 & 0,0776 & 0,1765 & 0,1008 & 0,2240 \\
\hline
\end{tabular}


FISCHER et al.

Tabela 7 - Valores das ordenadas do periodograma relativos à probabilidade de estar descansando segundo os períodos experimentais nas 12 primeiras freqüências de Fourier

Table 7 - Periodogram ordinates values for probability of being idling according to experimental periods at the first twelve Fourier frequencies

\begin{tabular}{|c|c|c|c|c|c|c|}
\hline \multirow{4}{*}{$\begin{array}{l}\text { Freqüência } \\
\text { Frequency }\end{array}$} & \multicolumn{6}{|c|}{$\begin{array}{l}\text { Descanso } \\
\text { Idling }\end{array}$} \\
\hline & \multicolumn{6}{|c|}{$\begin{array}{c}\text { Períodos experimentais } \\
\text { Experimental periods }\end{array}$} \\
\hline & Outubro & Novembro & Dezembro & Janeiro & Fevereiro & Março/abril \\
\hline & October & November & December & January & February & March/April \\
\hline 1 & 0,4501 & 1,3070 & 1,2801 & 1,1705 & 2,7386 & 1,1602 \\
\hline 2 & 0,1188 & 0,0624 & 0,3018 & 0,2686 & 0,3141 & 0,1399 \\
\hline 3 & 0,1979 & 0,1169 & 0,0398 & 0,0863 & 0,0504 & 0,1683 \\
\hline 4 & 0,4876 & 0,2692 & 0,4918 & 0,0359 & 0,5307 & 0,4449 \\
\hline 5 & 0,0099 & 0,0100 & 0,0180 & 0,0478 & 0,2222 & 0,0026 \\
\hline 6 & 0,4532 & 0,2346 & 0,4978 & 0,2534 & 0,1025 & 0,2718 \\
\hline 7 & 0,0785 & 0,1352 & 0,4786 & 0,3909 & 0,2961 & 0,4343 \\
\hline 8 & 0,0955 & 0,0108 & 0,0475 & 0,0170 & 0,1512 & 0,0599 \\
\hline 9 & 0,2597 & 0,0978 & 0,2951 & 0,1572 & 0,1481 & 0,1042 \\
\hline 10 & 0,0505 & 0,2895 & 0,0402 & 0,0914 & 0,6152 & 0,2525 \\
\hline 11 & 0,0263 & 0,1446 & 0,0134 & 0,0124 & 0,0818 & 0,2816 \\
\hline 12 & 0,1318 & 0,0423 & 0,0416 & 0,1083 & 0,0624 & 0,0312 \\
\hline
\end{tabular}

em cinco das 12 primeiras freqüências de Fourier: freqüências 1, 2, 3, 4 e 6 (Tabela 2), relacionadas principalmente às diferenças de amplitude das funções trigonométricas ajustadas às curvas de distribuição nictemeral das probabilidades de estar no estado de descanso (Tabela 8).

A evolução nictemeral das possibilidades de mudar de estado apresentou diferenças significativas entre os períodos de medidas e entre animais (Tabe- las 9 e 10). Pode-se verificar que as probabilidades de transição de estado envolvendo o estado de ingestão apresentaram diferenças significativas em um número superior de freqüências que aquelas que não envolveram o estado de ingestão. O número de freqüências em que o efeito de período se mostrou significativo foi inferior para as probabilidades de mudança do estado ingestivo para outros estados em relação às probabilidades de mudança de outros estados para o ingestivo

Tabela 8 - Valores das ordenadas do periodograma relativo à probabilidade de estar descansando segundo os animais experimentais nas 12 primeiras freqüências de Fourier

Table 8 - Periodogram ordinates values for probability of being idling according to experimental animals at the first twelve Fourier frequencies

\begin{tabular}{|c|c|c|c|c|c|c|c|c|c|}
\hline \multirow[b]{3}{*}{$\begin{array}{l}\text { Freqüência } \\
\text { Frequency }\end{array}$} & \multicolumn{8}{|c|}{$\begin{array}{l}\text { Descanso } \\
\text { Idling }\end{array}$} & \multirow[b]{3}{*}{9} \\
\hline & \multicolumn{8}{|c|}{$\begin{array}{c}\text { Animais experimentais } \\
\text { Experimental animals }\end{array}$} & \\
\hline & 1 & 2 & 3 & 4 & 5 & 6 & 7 & 8 & \\
\hline 1 & 1,3151 & 1,9902 & 2,3438 & 0,2758 & 1,2558 & 1,8655 & 1,2027 & 0,8276 & 1,7155 \\
\hline 2 & 0,1961 & 0,2812 & 0,1009 & 0,1698 & 0,3277 & 0,3410 & 0,0617 & 0,1721 & 0,3166 \\
\hline 3 & 0,3671 & 0,0430 & 0,0049 & 0,2586 & 0,2088 & 0,3137 & 0,3222 & 0,8393 & 0,0646 \\
\hline 4 & 0,3602 & 0,3438 & 0,2100 & 0,3513 & 0,4152 & 0,6086 & 0,3504 & 0,8402 & 0,2566 \\
\hline 5 & 0,0065 & 0,0532 & 0,0518 & 0,0157 & 0,0206 & 0,0751 & 0,0599 & 0,0017 & 0,1016 \\
\hline 6 & 0,2697 & 0,5638 & 0,0330 & 0,1837 & 0,9008 & 0,4721 & 0,2258 & 0,5446 & 0,0291 \\
\hline 7 & 0,2772 & 0,7912 & 0,1590 & 0,2265 & 0,3322 & 0,3656 & 0,0900 & 0,1595 & 0,4010 \\
\hline 8 & 0,5502 & 0,1581 & 0,0513 & 0,0942 & 0,0403 & 0,0361 & 0,0493 & 0,0397 & 0,0958 \\
\hline 9 & 0,0221 & 0,0476 & 0,0065 & 04632 & 0,1592 & 0,1911 & 0,5176 & 0,0988 & 0,0650 \\
\hline 10 & 0,1849 & 0,3664 & 0,1931 & 0,1480 & 0,5425 & 0,3702 & 0,1036 & 0,0196 & 0,0924 \\
\hline 11 & 0,3334 & 0,1247 & 0,0433 & 0,1472 & 0,0749 & 0,1579 & 0,0284 & 0,0569 & 0,0318 \\
\hline 12 & 0,1647 & 0,1499 & 0,0009 & 0,1006 & 0,2897 & 0,1563 & 0,0955 & 0,0930 & 0,0646 \\
\hline
\end{tabular}


Rev. bras. zootec.

1829

Tabela 9 - Valores das probabilidades mínimas de rejeição da hipótese de nulidade dos efeitos período e animal obtidos a partir da análise da variância das séries de probabilidades transição de estado, modificadas pela transformação finita de Fourier em cada freqüência

Table 9 - Minimal probabilities values of null hypothesis rejection of no animal or period effect obtained from variance analysis of transition state probablilities series modified throught finite Fourier transformation at each frequency

\begin{tabular}{|c|c|c|c|c|c|c|}
\hline \multirow[b]{3}{*}{$\begin{array}{l}\text { Freqüência } \\
\text { Frequency }\end{array}$} & \multicolumn{6}{|c|}{$\begin{array}{c}\text { Valores de } \mathrm{P} \\
P \text { values }\end{array}$} \\
\hline & \multicolumn{2}{|c|}{$\begin{array}{c}\text { Ingerindo-Descansando } \\
\text { Eating-Idling }\end{array}$} & \multicolumn{2}{|c|}{$\begin{array}{l}\text { Ingerindo-Ruminando } \\
\text { Eating-Ruminating }\end{array}$} & \multicolumn{2}{|c|}{$\begin{array}{c}\text { Ruminando-Ingerindo } \\
\text { Ruminating-Idling }\end{array}$} \\
\hline & $\begin{array}{l}\text { Animal } \\
\text { Animal }\end{array}$ & $\begin{array}{l}\text { Período } \\
\text { Period }\end{array}$ & $\begin{array}{l}\text { Animal } \\
\text { Animal }\end{array}$ & $\begin{array}{c}\text { Período } \\
\text { Period }\end{array}$ & $\begin{array}{l}\text { Animal } \\
\text { Animal }\end{array}$ & $\begin{array}{c}\text { Período } \\
\text { Period }\end{array}$ \\
\hline$\overline{0^{1}}$ & 0,001 & 0,292 & 0,001 & 0,019 & 0,001 & 0,294 \\
\hline 1 & 0,001 & 0,021 & 0,001 & 0,485 & 0,004 & 0,208 \\
\hline 2 & 0,016 & 0,183 & 0,005 & 0,548 & 0,001 & 0,133 \\
\hline 3 & 0,001 & 0,071 & 0,014 & 0,643 & 0,090 & 0,007 \\
\hline 4 & 0,006 & 0,164 & 0,001 & 0,145 & 0,812 & 0,392 \\
\hline 5 & 0,056 & 0,013 & 0,015 & 0,909 & 0,570 & 0,070 \\
\hline 6 & 0,501 & 0,003 & 0,246 & 0,250 & 0,860 & 0,178 \\
\hline 7 & 0,528 & 0,153 & 0,198 & 0,181 & 0,891 & 0,001 \\
\hline 8 & 0,021 & 0,623 & 0,001 & 0,959 & 0,028 & 0,086 \\
\hline 9 & 0,711 & 0,218 & 0,271 & 0,751 & 0,560 & 0,001 \\
\hline 10 & 0,001 & 0,190 & 0,001 & 0,439 & 0,087 & 0,162 \\
\hline 11 & 0,001 & 0,031 & 0,005 & 0,264 & 0,347 & 0,041 \\
\hline 12 & 0,511 & 0,123 & 0,187 & 0,269 & 0,520 & 0,031 \\
\hline
\end{tabular}

Tabela 10 - Valores das probabilidades mínimas de rejeição da hipótese de nulidade dos efeitos período e animal obtidos a partir da análise da variância das séries de probabilidades transição de estado, modificadas pela transformação finita de Fourier em cada freqüência

Table 10 - Minimal probabilities values of null hypothesis rejection of no animal or period effect obtained from variance analysis of transition state probablilities series modified throught finite Fourier transformation at each frequency

\begin{tabular}{|c|c|c|c|c|c|c|}
\hline \multirow[b]{3}{*}{$\begin{array}{l}\text { Freqüência } \\
\text { Frequency }\end{array}$} & \multicolumn{6}{|c|}{$\begin{array}{c}\text { Valores de } \mathrm{P} \\
P \text { values }\end{array}$} \\
\hline & \multicolumn{2}{|c|}{$\begin{array}{c}\text { Descansando-Ingerindo } \\
\text { Idling-Eating }\end{array}$} & \multicolumn{2}{|c|}{$\begin{array}{c}\text { Descansando-Ruminando } \\
\text { Idling-Ruminating } \\
\end{array}$} & \multicolumn{2}{|c|}{$\begin{array}{c}\text { Ruminando-Descansando } \\
\text { Ruminating-Idling }\end{array}$} \\
\hline & $\begin{array}{l}\text { Animal } \\
\text { Animal }\end{array}$ & $\begin{array}{c}\text { Período } \\
\text { Period }\end{array}$ & $\begin{array}{l}\text { Animal } \\
\text { Animal }\end{array}$ & $\begin{array}{c}\text { Período } \\
\text { Period }\end{array}$ & $\begin{array}{l}\text { Animal } \\
\text { Animal }\end{array}$ & $\begin{array}{l}\text { Período } \\
\text { Period }\end{array}$ \\
\hline$\overline{0^{1}}$ & 0,001 & 0,040 & 0,010 & 0,018 & 0,176 & 0,168 \\
\hline 1 & 0,001 & 0,020 & 0,002 & 0,410 & 0,001 & 0,092 \\
\hline 2 & 0,072 & 0,210 & 0,006 & 0,130 & 0,015 & 0,121 \\
\hline 3 & 0,041 & 0,097 & 0,060 & 0,009 & 0,001 & 0,009 \\
\hline 4 & 0,001 & 0,173 & 0,080 & 0,111 & 0,106 & 0,041 \\
\hline 5 & 0,046 & 0,022 & 0,989 & 0,170 & 0,164 & 0,023 \\
\hline 6 & 0,081 & 0,001 & 0,056 & 0,087 & 0,066 & 0,165 \\
\hline 7 & 0,014 & 0,001 & 0,161 & 0,029 & 0,043 & 0,153 \\
\hline 8 & 0,564 & 0,001 & 0,006 & 0,257 & 0,870 & 0,754 \\
\hline 9 & 0,508 & 0,713 & 0,293 & 0,144 & 0,220 & 0,968 \\
\hline 10 & 0,597 & 0,081 & 0,173 & 0,631 & 0,538 & 0,076 \\
\hline 11 & 0,025 & 0,009 & 0,473 & 0,287 & 0,203 & 0,982 \\
\hline 12 & 0,432 & 0,450 & 0,704 & 0,559 & 0,198 & 0,875 \\
\hline
\end{tabular}


(4 x 11), ocorrendo o mesmo fenômeno em relação ao efeito animal $(9 \times 15)$.

Ocorreu o inverso em relação à ruminação e ao repouso, o que provavelmente está relacionado ao impacto das práticas de manejo e dos fatores ligados ao ambiente deste estudo sobre a apreensão e ingestão do alimento, que se modificaram no decorrer do experimento, como o comprimento do dia. Por outro lado, a própria natureza do ato de ingestão possivelmente contribuiu para essas diferenças, destacando-se a sua duração e intensidade mais variável entre os momentos do dia e entre os períodos e a sua maior concentração em determinados momentos do nictêmero em relação às atividades de ruminação e descanso (DULPHY e FAVERDIN, 1987; DE BOEVER, 1991). Os mesmos autores destacam também a grande variabilidade interindividual quanto aos parâmetros do comportamento ingestivo.

A inspeção visual dos gráficos da evolução nictemeral das probabilidades (FISCHER et al., 2000) indicou a existência de variação do valor das probabilidades, segundo o momento do dia em que eram avaliadas. O uso da TFF e do teste de White noisy (SAS, 1989) revelaram que o padrão de variação destas probabilidades não é aleatório, mas obedece a determinada ritmicidade. Os resultados obtidos por meio da análise da variância da TFF permitiram detectar a ocorrência de diferenças entre os períodos de medida do comportamento ingestivo e entre os animais relacionadas à amplitude ou à diferença de fase da distribuição nictemeral das séries de probabilidades de transição de estado. A análise de periodogramas e diagramas de fase permitiu atribuir essas diferenças à amplitude e/ou defasagem da ocorrência do pico de probabilidade em relação ao início do registro do comportamento ingestivo, de maneira mais sensível e precisa que a análise visual dos gráficos de distribuição nictemeral da distribuição das probabilidades de transição de estado.

ROOK e PENNING (1991) testaram três modelos matemáticos para o cálculo das probabilidades de transição de estado, o multinomial, os processos de Markov e um modelo, chamado de dependente do tempo. Neste último modelo, as probabilidades de transição de estado foram calculadas considerando o efeito de um número crescente de 1 a 10 períodos de cinco minutos em determinado estado prévio à mudança de estado. Neste estudo, os autores concluíram que tanto o modelo com processos de Markov, que só considera o estado prévio imediatamente anterior ao da mudança de estado, como aquele dependente do tempo apresentaram respostas semelhantes, e ambos foram superiores ao modelo multinomial, que não considera os estados prévios na descrição e na simulação do comportamento ingestivo dos animais. Os próprios autores reconheceram, no entanto, que uma das limitações no uso dos processos de Markov é que as probabilidades de transição de estado são constantes. Os resultados obtidos no presente trabalho originam-se de um cálculo de probabilidades distinto daquele apresentado por ROOK E PENNING (1991), e a dependência do tempo refere-se ao momento do dia em que as probabilidades foram calculadas, e não ao período de tempo gasto em determinada atividade anterior à mudança de estado. De acordo com estes resultados, as probabilidades de transição de estado não são constantes, podendo apresentar variação acentuada, conforme o momento do dia em que são medidas.

\section{Conclusões}

A aplicação do cálculo de probabilidades ao estudo do comportamento ingestivo de ruminantes e posterior análise da variância sobre os dados de séries de probabilidades modificados segundo a aplicação da transformação finita de Fourier permitem a descrição do comportamento animal durante o nictêmero, considerando as peculiaridades dos ruminantes, como a concentração das atividades ingestivas em determinados momentos do dia, a influência do arraçoamento sobre a motivação dos animais em iniciar ou continuar uma refeição e o ritmo circadiano das atividades de ingestão e ruminação.

Nas condições do presente trabalho, recomenda-se considerar os efeitos do momento do dia, das diferenças interindividuais e do período do ano no cálculo dos valores médios de probabilidades de transição de estado dependentes do tempo.

\section{Referências Bibliográficas}

BEAUCHEMIN, K.A., KACHANOSKI, R.G. SCHAALJE, G.B. et al. 1990. Characterizing rumination patterns of dairy cows using spectral analysis. J. Anim. Sci., 68:3163-3170.

BRILLINGER, D.R. 1973. The analysis of time series collected in an experimental design. In: KRISHNAIAH, P.R. (Ed.). Multivariate analysis III. London: Academic Press. p.243-256. COLGAN, P. 1978. Quantitative ethology. New York, Wiley. DESWYSEN, A.G., DUTILLEUL, P., ELLIS, W.C. 1989. Quantitative analysis of nycterohemeral eating and ruminating patterns in heifers with different voluntary intakes and effects of monensin. J. Anim. Sci., 67:2751-2761.

DESWYSEN, A.G., DUTILlEUL, P., GODFRIN, J.P. et al. 1993. Nycterohemeral eating and ruminating patterns in 
Rev. bras. zootec.

heifers fed grass or corn silages : analysis by finite Fourier transform. J. Anim. Sci., 71:2739-2747.

DE BOEVER, J.I. Roughage evaluation of maize and grass silage based on chewing activity measurements with cows. Gand, Bélgica, Universidade de Gand, 1991. 250p. PhD Thesis.

DULPHY, J.P., FAVERDIN, P. 1987. L'ingestion alimentaire chez les ruminants:modalités et phénomènes associés. Reprod. Nutr. Dévelop., Paris, 27(1B):129-155.

DUTILlEUL, P. 1990. Apport en analyse spectrale d'un périodogramme modifié et modélisation des séries chronologiques avec répetition en vue de leur comparaison en fréquence. Louvain-la-Neuve, Belgium, Catholic University of Louvain. Tese de Doutorado.

DUTILLEUL, P. 1997. Incorporating scale in study design: data analysis. In: PETERSON, D.L., PARKER, V.T. (Eds.) Ecological scale: theory and applications. New York: Columbia University Press. p.1-77.

DUTILLEUL, P., DESWYSEN, AG., FISCHER, V. et al. 2000. Time-dependent transition probabilities and the assessment of seasonal effects on within-day variations in chewing behavior of housed sheep. Applied Anim. Behavior Sci., 68:13-37.

FAGEN, R.M., YOUNG, D.Y. 1978. Temporal patterns of behaviors: Durations, intervals, latencies, and sequences. In: Quantitative ethology. COLGAN, P.W. (Ed.). New York: Wiley. p.79-114.

FISCHER, V. 1996. Efeito da pressão de pastejo, do fotoperíodo e da dieta sobre o comportamento ingestivo de ruminantes. Porto Alegre, RS, UFRGS, 1996. 233 p. Tese (Doutorado em Produção Animal) - Faculdade de Agronomia, Universidade Federal do Rio Grande do Sul.
FISCHER, V., DESWYSEN, A.G., AMOUCHE, E. et al. 1998a Efeitos da pressão de pastejo sobre o padrão nictemeral do comportamento ingestivo de ovinos em pastejo. R. Bras. Zootec., 27(1):164-170.

FISCHER, V., DESWYSEN, A.G.,DESPRES, L. et al. 1998b. Padrões nictemerais do comportamento ingestivo de ovinos. R. Soc. Bras. Zootec., 27(2):362-369.

FISCHER, V., DUTILLEUL, P., DESWYSEN, A.G. et al. 2000. A aplicação de probabilidades de transição de estado dependentes do tempo na análise quantitativa do comportamento ingestivo de ovinos - Parte I. Rev. bras. zootec., 29(6):1814-1822.

ROOK, A.J., PENNING, P.D. 1991. Stochastic models of grazing behavior in sheep. Appl. Anim. Behavior Sci., 32:167-177.

RUCKEBUSCH, Y. Recherches sur la régulation centrale du comportement alimentaire chez les ruminants. Lyon, França: Universidade de Lyon. 1963. 213p. PhD Thesis.

S.A.S. 1988. SAS/ETS User's guide, version 6 (1.ed.). SAS Inst., INC, Cary, NC

S.A.S. 1989. SAS/STAT User's Guide, version 6 (4.ed). SAS Inst., Cary, NC

SNEDECOR, G.W., COCHRAN, W.G. 1967. Statistical methods. 6.ed. Ames: Iowa State University Press. 199p.

ZINN, S.A., CHAPIN, L.T., TUCKER, H.A. 1983. Does photoperiod and time of feeding affect growth and eating patterns of heifers? J. Dairy Sci., 66(suppl. 1):217.

Recebido em: 30/04/99

Aceito em: 19/10/00 